\title{
Recovery aspects in a case of incomplet paraplegia with a T11 neurological level, acute postischemia due to a hemorrhageic shock through an aorto-sigmoid fistula and communicative anastomotic pseudoaneurysm - Case report
}

Mihaela Mandu ${ }^{1}$, Cristinel Dumitru Badiu ${ }^{1,2}$, Doroteea Teoibaș-Șerban ${ }^{1,2}$, Raluca Petcu ${ }^{1}$, Anca Chiralidis ${ }^{1}$, Andreea Ioniță ${ }^{1}$, Mihai Băilă ${ }^{1}$, Razvan Oprea ${ }^{1}$, Gelu Onose ${ }^{1,2}$

Corresponding author: Mihaela Mandu, E-mail address: mihaelamandu37@yahoo.com

${ }^{1}$ The Teaching Emergency Hospital "Bagdasar-Arseni” (TEHBA), Bucharest, Romania ${ }^{2}$ The University of Medicine and Pharmacy ”Carol Davila” (UMPCD), Bucharest, Romania

\begin{abstract}
Introduction:The pseudo aneurysm is an accumulation of blood between the muscle and the adventitia of an artery, while a genuine aneurysm is "a permanent and localized dilation which determines an increase of more than 50\% in the normal diameter of the respective vessel" [1], [2]. Aneurysms are more common in the aorta and most frequently occur in male patients aged between 6585 years, representing 1-3\% of the total death causes in this group of age. [3], [4].

Material and methods:The paper presents the case of a 53-year old patient with incomplete paraplegia, AIS/Frankel D with a T11 neurologic level, acute post-ischemia due to a hemorrhagic shock. The patient was diagnosed in 2017 with ruptured right iliac aneurysm which required surgery. A right iliac exograft was inserted, which later became infected, thus requiring multiple surgical interventions, the patient remaining a chronic carrier of Enterococus Faecium. Subsequently, multiple pseudo aneurysms begin appear in the right and left iliac artery and aortic bifurcation. Multiple surgical inetervention ware performed. In January 2018, the patient presented himself with an emergency to the hospital, with a hemorrhagic shock. He was diagnosed with anastomotic pseudo aneurysm which communicated through a very long path with a fistula at the level of the sigma, showing small amounts of a periprosthetic collection with purulent aspect. Surgery is reinitiated by insertion of an axillo-bifemoral bypass and Hartman resection with colostomy. Another important element in the pathological history of the patient is operated pulmonary neoplasm with hepatic and bone metastases. The patient was clinically and functionally evaluated, according the standardized protocols implemented in our clinic, through the assessment scales (AIS, FIM, QoL-Quality of Life, Asworth, Penn, FAC, WISCI II) and also paraclinically, in order to evaluate his biological reserve and his bearing availability of the recovery program.

Results: The patient presented a slowly favorable evolution (slowed down by his multiple above mentioned comorbidities) from an algo-dysfunctional point of view, with the improvement of the walking program and the increase of muscle force and individual autonomy.

Conclusion: Although aortic aneurysms are common between 65-85 years of age, they can also appear at younger ages. The aortoenteric fistula is a rare cause of massive gastrointestinal bleeding, many of them leading to the death of the patient before presentation to the doctor. Although medullary ischemia can lead to neurologic deficit of the paraplegic type, it can be corrected through a complex recovery program.
\end{abstract}

Key words: pseudoaneurysm, paraplegia, incomplete, fistula, ischemia

\section{Introduction}

Pseudoaneurysm is a collection of blood between the tunica media and the tunica adventitia of an artery, while a real aneurysm is "a permanent, localized dilation which determines an increase by more than $50 \%$ of the normal diameter of the given vessel"'[1],[2]. Aneurysms are more common in the aorta and often occur in male patients aged between $65-85$, which represents $1-3 \%$ of all the causes of death in this group of age [3],[4]. The aorto-enteric fistula is a rare cause of massive gastrointestinal bleeding, which could have catastrophic consequences through the hemorrhagic shock caused, many such fistulae leading to the death of the patient [5],[6]. There may be medullary ischemia due to the massive loss of blood, which may be presented through a wide range of clinical manifestations: pain, motor deficit, sometimes the impairment of the sphincter control.
Materials and methods The present paper presents the case of a patient with incomplete paraplegia AIS/Frankel D with an acute post ischemia T11 neurologic level due to a hemorrhagic shock. In 2017, he was diagnosed with ruptured right iliac aneurysm for which he underwent surgery, a right iliac exograft was inserted which became infected, which required several surgical interventions, while the patient remained a chronic carrier of Enterococcus Faecium. Subsequently, there was a non-infected pseudoaneurysm in the right iliac fossa, which required the surgical re-intervention with the resection of the aortic bifurcation and left aorto-iliac prosthetic bypass. In January 2018, the patient comes for an emergency to the hospital with a hemorrhagic shock and diagnosed with anastomotic pseudo-aneurysm which communicates through a pretty long pathway with a fistula in the right sigma, with a small amount of periprosthetic collection with a purulent aspect. 
Surgical re-intervention is performed by insertion of an axillo-bifemoral by-pass and Hartman resection with colostomy.

The objective examination upon admission revealed: poorly represented adipose connective tissue (marked weight loss: 10 kilos in the last two months); muscular system hypotone, hypokinetic; abdominal scar on the medial line supraumbilical up to the level of the xiphoid process; a colostomy bag (Fig.1); multiple scars on the right hemibody (Fig.2); second degree pressure sores on the left buttock-sacrum and small dimension sores on the right buttock (Fig.3).

NMAK examination revealed : conscious, cooperative, temporally and spatially oriented, auto and allopsychically oriented ; motor deficit of the incomplete paraplegia type - bilateral lower limbs (LLs); reduced osteotendinous reflexes - bilateral lower limb; sensitivity disturbances- dorsal part of the left foot and the toes of the left foot; Right lumbosacral pain; Crackling noises at mobilization of both knees; Motor deficit at thigh flexion 4/5 left of lower limb and plantar dorsiflexion $0 / 5$ of the left lower limb; Muscular force deficit at left calf extension 3/5.

From a functional point of view the patient keeps resting in bed and tolerates posturing into the wheel chair for short-medium periods of time (otherwise non-recommended, given the previously mentioned pressure sores).

The patient was clinically and functionally assessed, according to the standardized protocols implemented in our clinic by means of the assessment grading scales: AIS (American Spinal Injury Association Impairment Scale), FIM (Functional Independence Measure), QoL (Quality of Life) (Flanagan completed by Burckhard), Asworth si Penn, FAC (Functional Ambulation Category), WISCI II (Walking Index for Spinal Cord Injury).

The patient was paraclinically examined in order to evaluate his biological reserve and his availability in bearing the recovery program. To this purpose, both laboratory and imaging investigations have been used. The laboratory investigations releaved: moderate hypoalbuminemia, moderate hyperglycemia, moderate hypokalemia, moderate , moderate normocytic and normochromic anaemia, moderate inflammatory biological syndrome.

During the hospital stay, the patient underwent a complex recovery program which included: treatment with medication (oral and then injectable anticoagulants, platelet antiaggregants, cardiac canal inhibitors, hypolipemic drugs, gastric protectors, oral iron supplements, venous vascular wall protectors, local antibiotics, urinary antiseptics, antihemorrhoidal suppositories, laxatives, sleeping pills, morphine-derivative painkillers) and physical treatment (kinesiotherapy).

\section{RESULTS}

After a complex neurorecovery program undertaken by a multidisciplinary team formed by doctors, kinesiotherapists, medium healthcare and paramedical personnel, the patient displayed a slowly favorable evolution (hardened by his multiple associated diseases due to the above-mentioned comorbidities) from a pain-dysfunctional point of view with the improvement of the walking program and the increase of muscle force and self-autonomy.

\section{CONCLUSION}

Although aortic aneurysms are common between 6585 years of age, they can also appear at younger ages. The aorto-enteric fistula is a rare cause of massive gastrointestinal bleeding, many of them leading to the death of the patient before presentation to the doctor. Although medullary ischemia can lead to neurologic deficit of the paraplegic type, it can be corrected through a complex recovery program.

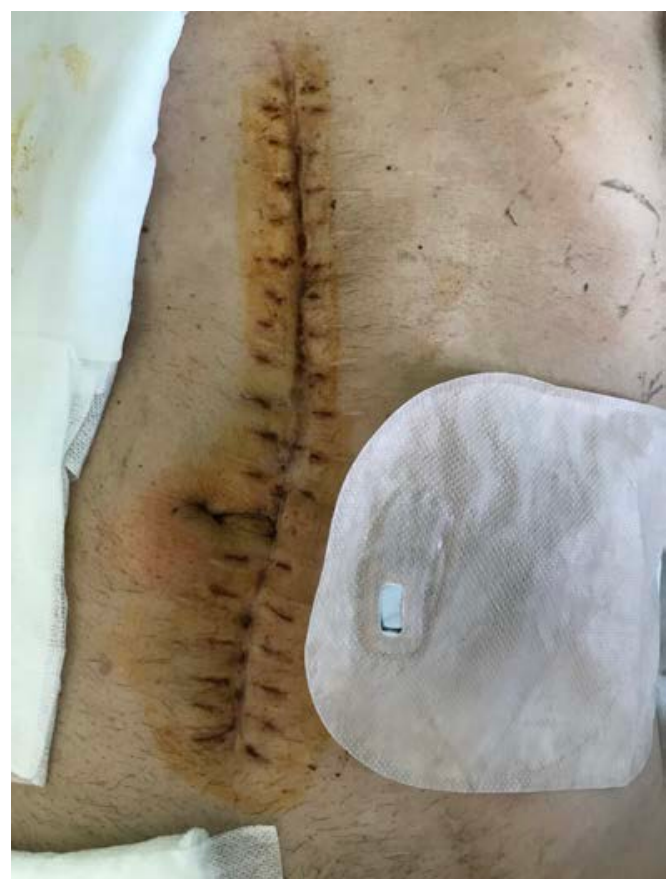

FIGURE 1: Abdominal scar on the medial line supraumbilical up to the level of the xiphoid process. A colostomy bag 


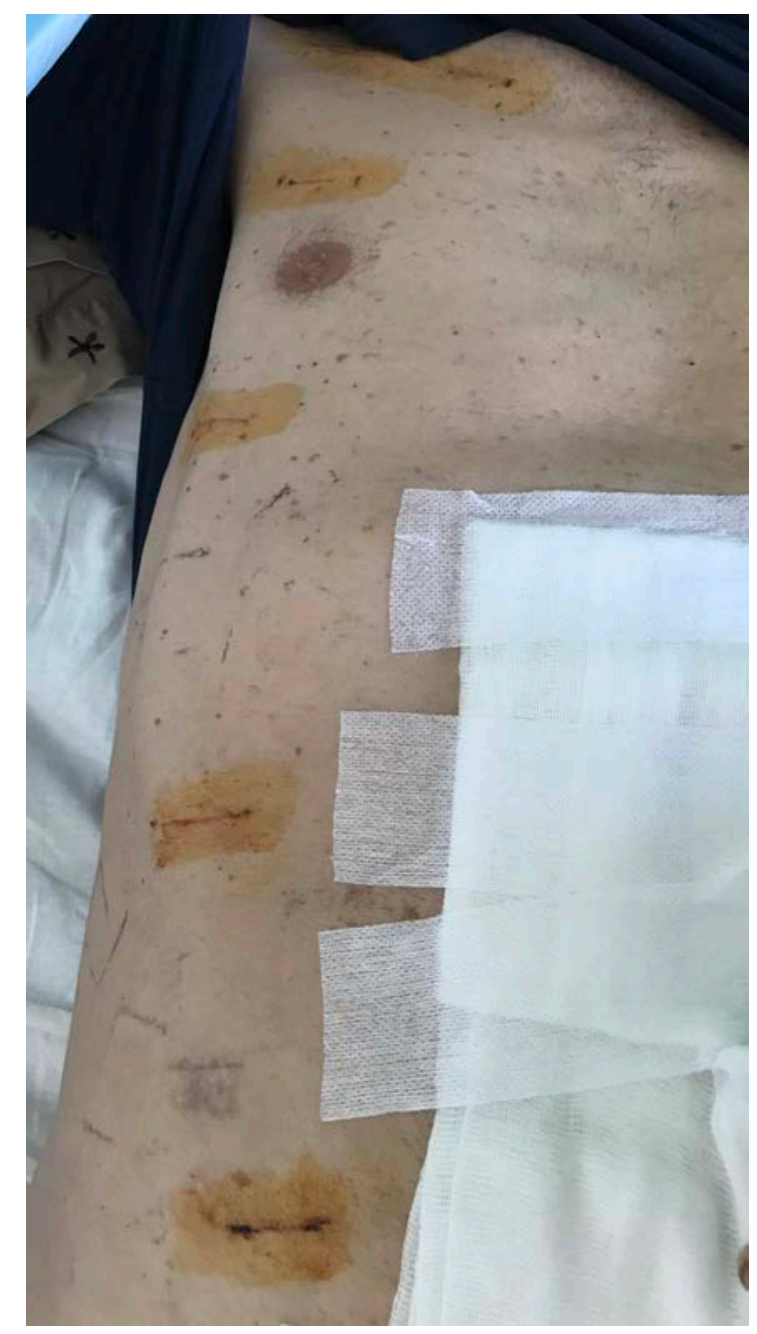

FIGURE 2: Multiple scars on the right hemibody

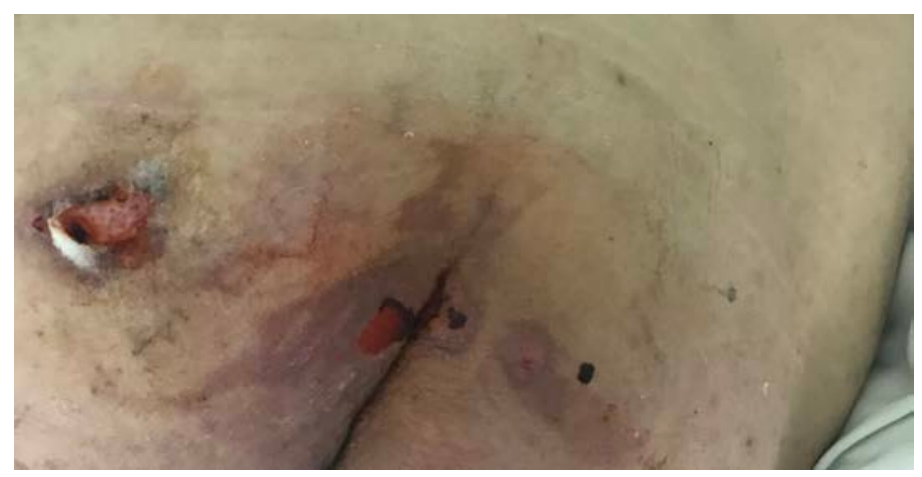

FIGURE 3: Second degree pressure sores on the left buttock-sacrum and small dimension sores on the right buttock

\section{Bibliography}

1. Donna D'Souza, False Aneurysm, Radiopedia, https://radiopaedia.org/articles/false-aneurysm;

2. M. Beuran, "Chirurgie pentru studenti”,pag 72, Editura Ilex, Bucuresti, 2013;

3. Saum A Rahimi, Abdominal Aortic Aneurysm, Medscape.com, Aug 16, 2017;

4. N. Sakalihasan, R.Limet, O.D.Defawe, Abdominal aortic aneurysm, The Lancet, Volume 365, Issue 9470, 30 April-6 May 2005, Pages 1577-1589;

5. Y.Ihama, T.Miyazaki, C.Fuke, Y.Ihama, R.Matayoshi, H.Kohatsu, F.Kinjo, An autopsy case of a primary aortoenteric fistula: A pitfall of the endoscopic diagnosis, World J Gastroenterol. 2008 Aug 7; 14(29): 4701-4704;

6. Lee W, Jung CM, Cho EH, Ryu DR, Choi D, Kim J, Primary aortoenteric fistula to the sigmoid colon in association with intra-abdominal abscess, Korean J Gastroenterol. 2014 Apr;63(4):239-43; 